were similar $(p>0.05)$. There were no significant differences in the incidence of MACE between two groups.

Conclusion ACS patients with loading dose clopidogrel combined with simvastatin or fluvastatin could decrease the MACE, the results in two groups are similar. Neither simvastatin with clopidogrel nor fluvastatin with clopidogrel decreases the platelet activity of clopidogrel.

\section{e0631 OBSTRUCTIVE SLEEP APNOEA SYNDROME IS ASSOCIATED WITH THE INCREASED RISK OF LOW-ANTIPLATELET RESPONSE OF CLOPIDOGREL IN PATIENTS WITH UNSTABLE ANGINA}

doi:10.1136/hrt.2010.208967.631

Fu Xianghua, Li Shiqiang, Wang Oian, Gu Xinshun, Wang Yanbo, Wang Xuechao, Wu Weili, Xue Ling. The Second Hospital of Hebei Medical University

Objective To address the relationship between low antiplatelet response of clopidogrel and Obstructive Sleep Apnoea Syndrome (OSAS) in patients with unstable angina pectoris.

Methods Total of 112 patients hospitalised with unstable angina pectoris from February 2008 to December 2009 were enrolled in this randomised consecutive study. All patients accepted routine treatment including clopidogrel, aspirin, low molecular weight heparin daily. Platelet aggregation (PAR) parameters were measured on samples obtained at baseline and $2 \mathrm{nd}$, 4th, 6th day. All patients were examined for the presence of sleep-disordered breath into 4 quartiles by AponeaLink. The concentration serum adrenaline and norepinephrine were measured in the morning at 06:00 after the sleep study. Result There were no significant differences in the baseline data in all 4 quartiles. However, there was a significant differences in the number of diabetes patients in the first quartibesity, heale $(p=0.0038)$ compared with other quartiles. At day 2 PAR were inhibited to $63.91 \%$ of baseline $(p<0.01)$ and $88.38 \%(p>0.05)$ of baselinctively, in the first quartile. At each of these time points, platelet activity was significantly higher than in patients in other quartiles. At day 6 platelet aggregation were reduced to $32.37 \%$, and $29.75 \%$ of baseline respectively in group 2 through 4 ( $p<0.01$ for all). PAR was reduced significantly in patients in the second through fourth quartiles at day 6 , but, it showed a lower reduction in the first quartile $(p>0.05)$. Compared with that in thebidity of OSAS in the second and third were $25.0 \%$ and $14.3 \%(p<0.05)$, only $3.6 \%$ in the fourth group $(\mathrm{p}<0.01)$. Meanwhile, the concentration first group $(60.7 \%)$, the mor $\mathrm{n}$ of serum adrenaline and nine were higher in the first quartile than others $(p<0.05)$.

Conclusion OSAS is aicator of low clopidogrel response in unstable angina patients, and higher concentration of epinephrine and norepinephrine in OSAS pa reliable inpatients plaorepinephry a more important role in this situation.

\section{e0632 A RANDOMISED COMPARISON STUDY OF RECOMBINANT STAPHYLOKINASE VS RECOMBINANT TISSUE-TYPE TISSUE PLASMINOGEN ACTIVATOR FOR SAFETY AND CORONARY ARTERY PATENCY IN PATIENTS WITH ACUTE MYOCARDIAL INFARCTION}

doi:10.1136/hrt.2010.208967.632

Fu Xianghua, Sun Jiaan, Liu Jun, Wei Yongyun, Fan Weize, Wang Xuechao, Wang Yanbo. The Second Hospital of Hebei Medical University

Objective To evaluate the efficacy and safety of r-SAK (recombinant staphylokinase) for acute ST-segment elevation myocardial infarction (STEMI).

Methods A total of 48 patients with acute STEMI randomised into r-SAK group and r-tPA group (each with 24 patients). In r-SAK group, $10 \mathrm{mg}$ r-SAK diluted up to $50 \mathrm{ml}$ with saline before administration, $2 \mathrm{mg}$ bolus over $2 \mathrm{~min}$, followed by an infusion of the remaining $8 \mathrm{mg}$ over $30 \mathrm{~min}$. While in r-tPA group, first $8 \mathrm{mg}$ bolus over $6 \mathrm{~min}$, then $42 \mathrm{mg}$ over a 90 -min period. A $75 \mathrm{U} / \mathrm{kg}$ heparin bolus was given as r-SAK or r-tPA was infusing for anti-coagulation treatment. CAG were performed at $90 \mathrm{~min}$ to confirm infarction location and IRA, stenosis was analysed by OCA, IRA flow was evaluated by TIMI grades, myocardial tissue reperfusion was assessed by TMPG. Acute complications and adverse events were recorded during 30 days after thrombolysis.

Results There was no significant difference in baseline data between $r-S A K$ and r-tPA group. There was no difference in IRA distribution between the two groups, the IRA repatency rate $(p=0.308)$, TIMI 3 flow $(p=0.355)$, myocardial tissue reperfusion $(p=0.530)$ in r-SAK group are slightly higher than those in r-tPA group, but the differences was not significant. The acute complications during 30-day period after thrombolysis, include allergic reaction $(p=0.317)$, serious arrhythmias $(p=0.775)$, heart failure $(p=0.530)$, cardiac shock $(p=1.000)$, IRA re-occluded $(p=0.555)$, postinfarction angina $(p=0.734)$ and death $(p=0.317)$, have no significant difference between the two groups. The bleeding complications of r-SAK group were slightly less $(p=0.125)$. No statistic difference in adverse events was found between the two groups.

Conclusions r-SAK proved to be at least as effective as alteplase in inducing early coronary artery patency for STEMI with higher fibrin specificity than r-tPA, r-SAK, and less bleeding complications. The safety of r-SAK thrombolysis therapy is at about the same level of that of $\mathrm{r}-\mathrm{tPA}$, not associate with excess mortality and complications of arrhythmia, postinfarction angina and haemorrhage.

\section{E0633 THE EFFECT ON LEFT VENTRICULAR FUNCTION AND SAFETY OF HIGH MAINTENANCE DOSE OF CLOPIDOGREL IN PATIENTS WITH ACUTE ANTERIOR MYOCARDIAL INFARCTION UNDERGOING SELECTIVE PCI}

doi:10.1136/hrt.2010.208967.633

Fu Xianghua, Wang Cong, Wang Xuechao, Wang Yanbo, Gu Xinshun, Fan Weize, Wu Weili, Jiang Yunfa. The Second Hospital of Hebei Medical University

Objective To assess the beneficial efficacy and safety of the high maintenance dose of clopidogrel in acute anterior myocardial infarction (AMI) patients undergoing selective percutaneous coronary intervention (PCI).

Methods Fifty two patients were enrolled into this study. These cases were randomly divided into the high maintenance dose group $(n=26$, 14 males, $150 \mathrm{mg}$ clopidogrel per day) and the control group ( $\mathrm{n}=26$, 15 males, $75 \mathrm{mg}$ clopidogrel per day). OCA and TIMI Myocardial perfusion grading (TMPG) were used to analyse the lesion and reperfusion of the culprit vessel and myocardium. Record the information of patients in-hospital, in the 1 month and 6 months including the level of BNP, left ventricular ejection fraction (LVEF), the left ventricular peak ejection rate (LPER), the left ventricular peak filling rate (LPFR), the left ventricular time to peak ejection rate (LTPER) and left ventricular time to peak filling rate (LTPFR).

Result 1. The CTFC of the high maintenance dose group after PCI was smaller than the standard dose group. The percentage of TMPG 3 grade was higher in the high maintenance dose group. 2 . The left ventricular peak ejection rate (LPER), the left peak filling rate (LPFR) 6 months after PCI in the high maintenance dose group was higher than the control group. The left ventricular time to peak ejection rate (LTPER), left ventricular time to peak filling rate (LTPFR) 1 month after PCI in the high maintenance were lower than the control group. 3. There were less acute and subacute thrombosis cases in the high maintenance dose group than the standard dose group. There was no significant difference in haemorrhage events between two groups. 
Conclusion The high maintenance dose clopidogrel can improve cardiac function. There is potential benefit in increasing coronary blood flow and improving myocardium perfusion. High maintenance dose clopidogrel decreases the acute and subacute thrombosis but do not increase the haemorrhage events.

\section{e0634 THE RELATIONSHIP BETWEEN HYPOKALAEMIA AT THE EARLY STAGE OF ACUTE MYOCARDIAL INFARCTION AND MALIGNANT VENTRICULAR ARRHYTHMIA}

doi:10.1136/hrt.2010.208967.634

Fu Xianghua, Qi Peng, Wang Yanbo, Wang Yanbo, Li Shiqiang, Fan Weize, Jiang Yunfa. The Second Hospital of Hebei Medical University

Objective To investigate the relationship between hypokalaemia at the early stage of acute myocardial infarction (AMI) and malignant ventricular arrhythmia (MVA) as well as the features of hypokalaemia.

Methods Total of 302 patients were involved in this study and conformed to the following conditions: getting AMI primarily, onset was within 24 hours, accepted serum potassium test and Holter monitoring on admission, didn't use diuretics before, hyperthyroidism, diabetes, vomiting or diarrhoea resulted from gastrointestinal diseases. Relevant data including types of AMI, namely STEMI or NSTEMI; infarct sites of STEMI; time interval from onset of AMI to admission; whether or not hypokalaemia (serum potassium $\leq 3.5 \mathrm{mmol} / \mathrm{l}$ ) and MVA were recorded. The relationships between hypokalaemia and MVA, the time interval and hypokalaemia, types of AMI and hypokalaemia, infarct sites and hypokalaemia were analysed. SPSS 13.0 was used for statistical analysis. The categorical data was processed with chi-square test and $\mathrm{p}$ values below 0.05 were considered significant.

Results The incidence of hyokalemia for 24 patients within $3 \mathrm{~h}$ from onset of AMI to admission was $37.5 \%$. The incidence of MVA between the group with and without hypokalaemia had significant difference $(10.47 \%$ vs $3.36 \%, p<0.05)$. The incidence of hypokalaemia between the group within $3 \mathrm{~h}$ and group within $3 \mathrm{~h}$ to $24 \mathrm{~h}$ of time interval from onset of AMI to admission had significant difference $(37.5 \%$ vs $15.47 \%, p<0.05)$. There was no significant difference in incidence of hypokalaemia between the group of STEMI and NSTEMI (20.35\% vs $12.68 \%, p>0.05)$. There was no significant difference in incidence of hypokalaemia between groups with anterior wall AMI and non-anterior wall AMI $(25.88 \%$ vs $18.81 \%, \mathrm{p}>0.05)$.

Conclusion At the early stage of AMI, hypokalaemia is often present. MVA was close associated with hypokalaemia at the early stage of AMI, which indicated that hypokalaemia was a cause of death.

\section{e0635 THE EVALUATION TO THE EFFICACY AND SAFETY OF TIROFIBAN IN ACUTE CORONARY SYNDROME PATIENTS WITH CLOPIDOGREL RESISTANCE DURING PERCUTANEOUS CORONARY INTERVENTION}

doi:10.1136/hrt.2010.208967.635

Fu Xianghua, Gu Xinshun, Chai Qiaoying, Fan Weize, Zhang Jing, Hao Guozhen, Jiang Yunfa, Wu Weili, Li Shiqiang, Xue Ling. The Second Hospital of Hebei Medical University

Objective To assess the efficacy and safety of the tirofiban in acute coronary syndrome patients with clopidogrel resistance undergoing selective percutaneous coronary intervention (PCI).

Methods A total of 90 acute coronary syndrome patients with clopidogrel resistance were randomised into two groups, the high maintenance clopidogrel group (HMCG, $\mathrm{n}=50$ ) and the tirofiban group (TG, $\mathrm{n}=40$ ). All the patients underwent PCI after 7-10 day's medical treatment. Clinical information was collected. The platelet aggregation rate (PAR) were measured, and the markers of platelet activation, PAC-1 and CD62P were measured.

Result There was no significant difference in baseline data between two groups. The expression rate of CD62P and PAC-1 in HMCG and TG were higher than the normal control group, but no difference between clopidogrel group and the tirofiban group. After the medical treatment the expression rate of CD62P and PAC- 1 in TG is higher than that in HMCG $(p<0.05)$. At the time of $0.5 \mathrm{~h}$ after PCI, the expression rate of $\mathrm{CD} 62 \mathrm{P}$ and $\mathrm{PAC}-1$ is higher than that before $\mathrm{PCI}$ $(\mathrm{p}<0.05)$. Until $12 \mathrm{~h}$ after PCI the expression rate of CD62P and PAC-1 is dropped down to the level before PCI. There were less MACE cases in TG than that in HMCG in hospital $(p<0.05)$, but no significant difference in haemorrhage events between two groups. Conclusion $150 \mathrm{mg} / \mathrm{d}$ clopidogrel can inhibit the activation of platelet but $75 \mathrm{mg} / \mathrm{d}$ clopidogrel can't in patients of ACS with clopidogrel resistance. Tirofiban can decreases the MACE cases of patient with clopidogrel resistant during PCI but do not increase the haemorrhage events.

\section{e0636 THE RELATIONSHIP STUDY BETWEEN BNP LEVELS AND CK-MB, CTNI CONCENTRATIONS, THE DEGREE OF CORONARY ARTERY DISEASE, HEART FUNCTION IN PATIENTS WITH ST-SEGMENT ELEVATION ACUTE MYOCARDIAL INFARCTION}

doi:10.1136/hrt.2010.208967.636

Fu Xianghua, Wang Xuechao, Fan Weize, Wu Weili, Wang Yanbo, Gu Xinshun, Jiang Yunfa, Hao Guozhen. The Second Hospital of Hebei Medical University

Objective To analysis the relationship between BNP levels and CK$\mathrm{MB}, \mathrm{cTNI}$ concentrations, the degree of coronary artery disease and heart function in patients with acute ST-segment elevation myocardial infarction (STEMI).

Methods A total of 86 patients with AMI got intravenous thrombolysis within $6 \mathrm{~h}$ after myocardial infarction were divided into group A $(\mathrm{BNP}<100 \mathrm{pg} / \mathrm{ml})$, group B (BNP100-500 pg/ml), C group (BNP $>500 \mathrm{pg} / \mathrm{ml}$ ) according to the BNP peak level. The BNP level, $\mathrm{CK}-\mathrm{MB}$, and the cTNI peak concentrations within $24 \mathrm{~h}$ were examined. The heart function was examined by UCG within one week, cardioangiography was performed within 7-10 days after AMI, so that to evaluate the relationship between BNP levels and CK-MB, cTNI concentrations, the degree of coronary artery disease, heart function. Result There were no statistical differences in baseline data among A, B, C groups, the higher the BNP level. The higher the CK-MB level $(p<0.05)$, so is the cTNI peak level $(p<0.05)$. There was a significantly correlation between BNP peak levels and CK-MB, cTNI peak concentrations, while the higher the BNP level, the lower the LVEF was $(p<0.05)$, there was a significantly negatively correlation between BNP peak level and LVEF. Leaman coronary score show that scores in group $C$ are higher than that in $B$, A groups $(p<0.05)$ caused a positively significantly correlation between BNP peak level and leaman coronary score. There was increased trend of left ventricular end diastolic pressure (LVEDP) $(p<0.05)$. Spearman correlation analysis showed significantly correlation between BNP peak levels and CK-MB, cTNI, LVEF, LVEDP levels. The linear regression equation between BNP peak levels and LVEFs, cTNI levels were $\mathrm{Y}=0.5466-0.00015 \mathrm{X}, \mathrm{Y}=5.6314+0.0023 \mathrm{X}$ ( $\mathrm{p}$ all $<0.05$ ).

Conclusion The higher the BNP peak levels, the higher the CK-MB, cTNI levels and the lower the LVEF in acute myocardial infarction patients. The between BNP peak levels had positive with CK-MB, cTNI peak concentrations and the degree of coronary artery disease, negative correlation-ship with LVEF. 